\title{
Correlative Workflow in the Characterization of TiN Coatings in Cardiac Rhythm Management Devices
}

\author{
Joseph Favata ${ }^{1}$, Bahar Ahmadi ${ }^{1}$, Shahram Amini' ${ }^{2}$, and Sina Shahbazmohamadi ${ }^{1}$ \\ 1. REFINE Lab, University of Connecticut, Storrs, CT, USA \\ 2. Center for Coatings and Surface Solutions (CCSS), Johnson Matthey, West Chester, PA, USA
}

Implantable medical devices such as pacemakers, defibrillators, and neurostimulators require electrochemically active conductive electrodes with high surface area and low impedance to transfer electrical charge from the device to human tissue. The microstructure of these coatings such as titanium nitride (TiN) or iridium oxide $\left(\mathrm{IrO}_{2}\right)$ are of peak interest as it affects performance and any undue failure carries the risks associated with such failures [1-4]. The evaluation of these coated components is challenging given their length scale and the many techniques available for coating characterization including electron microscopy, optical microscopy, IR spectroscopy, and X-ray tomography, among others. But a three-dimensional and non-destructive evaluation method such as 3-D X-ray tomography presents a nearly ideal approach to evaluate these coatings. In short, entire components can be checked for coating irregularities just before product integration in a manufacturing setting. To validate the findings of the X-ray tomography for this application, FIB-SEM serial sectioning was used to probe distinct locations mapped from a high-resolution X-ray tomography by means of a correlative workflow.

Two specialty TiN coatings on flat Pt-Ir alloy substrates were the subjects of the study. One coating was deposited on a grit-blasted substrate, while the other was deposited on an untreated substrate (Sample A and Sample B, respectively in Figure 1). The coatings were imaged with a Zeiss Xradia 520 Versa (Pleasanton, CA) on a $160 \mathrm{keV} / 10 \mathrm{~W}$ X-ray beam setting at a $500 \mathrm{~nm}$ pixel size. The results of the two scans are shown in Figure 2 with distinct differences at the interface of the coating and substrate. Sample A shows splotches of contrast indicative of material differences and interface non-linearity, while Sample B shows no such features. Based on this information, Sample A would fail the validation necessary for product integration while Sample B would be considered nominal. But, further validation is needed to see if important information was missed.

Within Zeiss Atlas 5, the 3-D map was overlaid with 2-D SEM surface images and by feature registration with a fiducial mark (see Figure 2), the data sets' coordinate systems were then linked. The FIB-SEM serial sectioning was conducted on a Zeiss Crossbeam 340 (Oberkochen, Germany). The ion beam had an acceleration voltage of $30 \mathrm{kV}$ and a current of $700 \mathrm{pA}$. The electron beam imaged at a $5 \mathrm{kV}$ voltage and a $212 \mathrm{pA}$ current. The pixel size and slice thickness were both $20 \mathrm{~nm}$. The data sets were imported into Avizo Fire 9.1 where they were segmented and rendered. Figure 3 illustrates the differences observed in the Xray data sets at a much higher magnification and resolution. The FIB-SEM data shows that for Sample B, there were no features missed by the X-ray tomography and that the uniform interface existed on this length scale. The data for Sample A shows a much more detailed rendering of the void spacing in the coating as well as undulations in the interface. This correlation of data points illustrates that the X-ray tomography serves as an effective tool to detect irregularities in these specialty coatings. Because of the validation, this application will benefit from the cost-savings of only needing one verification technique for initial quality assurance testing, the time-savings of examining large-scale volumes of the components, and the fundamental nature of non-destructive evaluation. 
References:

[1] J. Amrani, Neuromodulation: Technology at the Neural Interface 17 (2014), p. 200.

[2] J. Henderson et al, Neuromodulation: Technology at the Neural Interface 9 (2006), p. 183.

[3] A. Coleman, T. Defrancesco and G. Chanoit, Journal of Veterinary Cardiology 14 (2012), p. 519.

[4] P. Nordbeck et al, Magnetic Resonance in Medicine 65 (2011), p. 44.
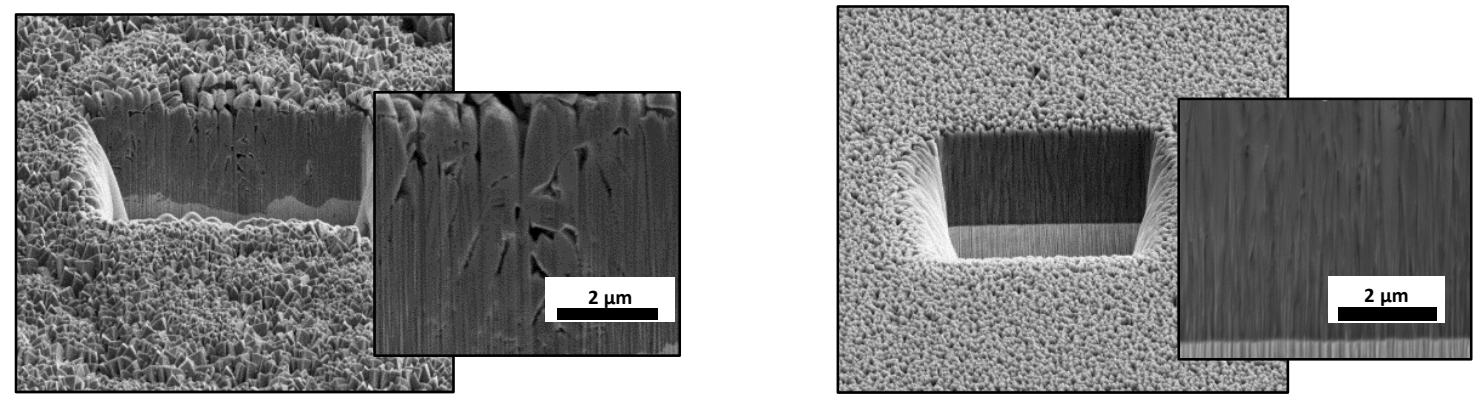

Figure 1. SE-SEM images of two TiN coatings on Pt-Ir alloys substrates selected for study; Sample A (left) and Sample B (right)
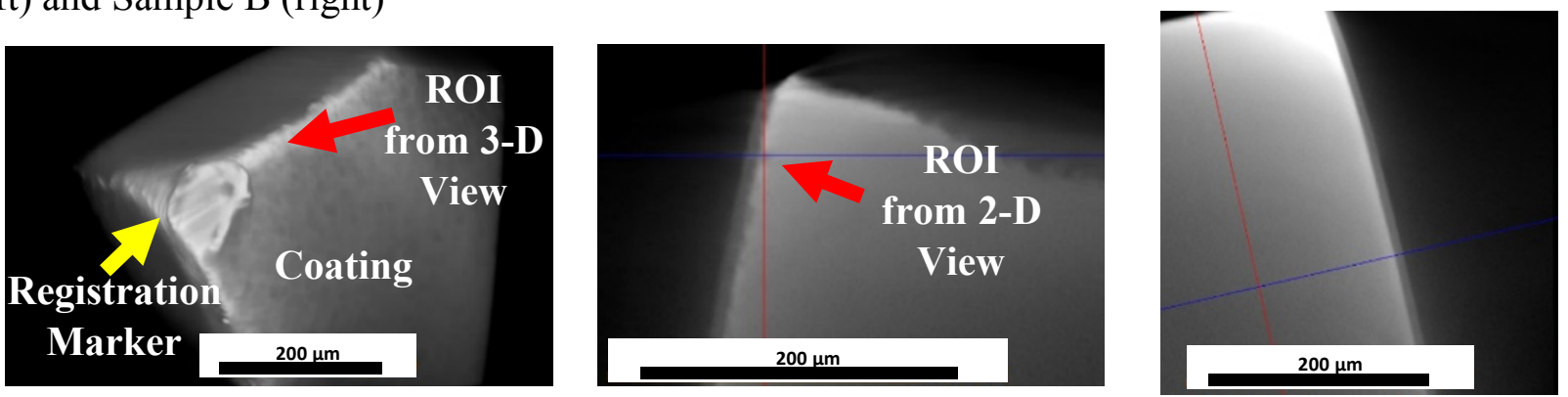

Figure 2. 3-D X-ray data set of Sample A (left) and digital cross-sections of Sample A and B (middle and right)
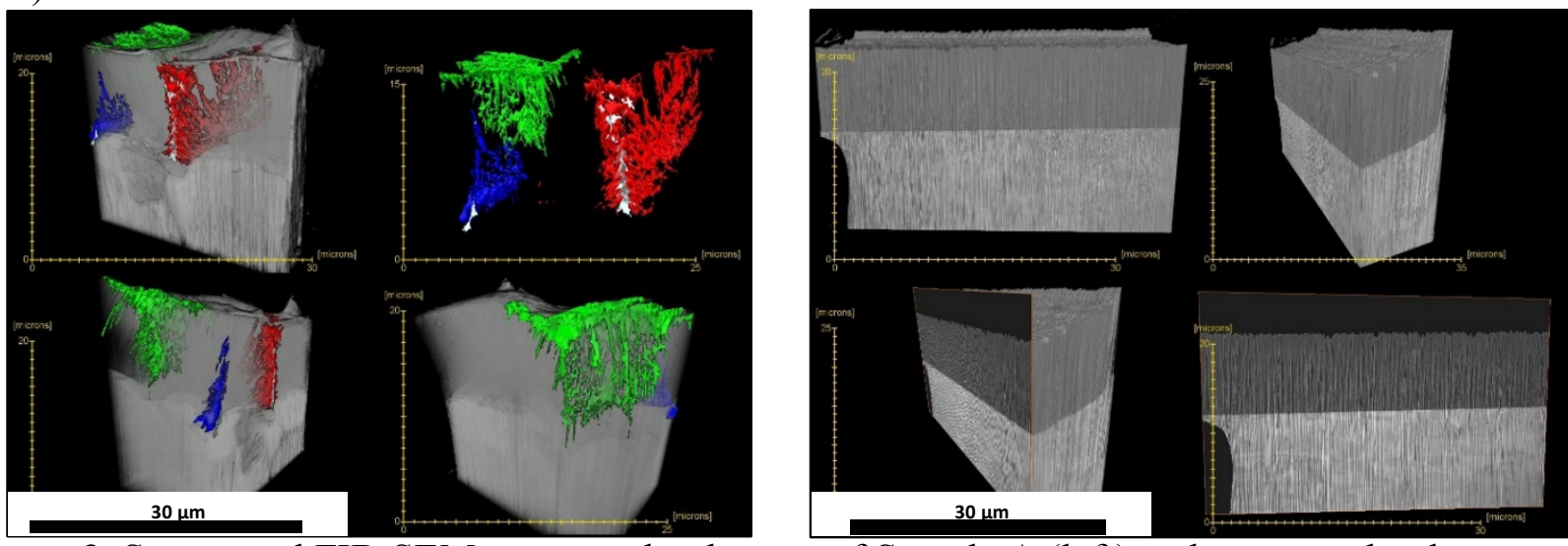

Figure 3. Segmented FIB-SEM tomography data set of Sample A (left) and tomography data set of Sample B (right) 